\title{
Patients with disturbances in consciousness as observed by clinical nurses: Analysis of nursing records
}

\author{
Ryoko Tsuchiya ${ }^{1}$, Kasumi Mikami ${ }^{2}$, Keiko Aidu ${ }^{2}$, Yoshiko Nishizawa ${ }^{2}$ \\ ${ }^{1}$ Hirosaki University School of Medicine \& Hospital, Aomori, Japan \\ ${ }^{2}$ Hirosaki University Graduate School of Health sciences, Aomori, Japan \\ Email: ryoko34@cc.hirosaki-u.ac.jp
}

Received 31 August 2013; revised 1 October 2013; accepted 15 October 2013

Copyright (c) 2013 Ryoko Tsuchiya et al. This is an open access article distributed under the Creative Commons Attribution License, which permits unrestricted use, distribution, and reproduction in any medium, provided the original work is properly cited.

\begin{abstract}
The objective of the present study was to discuss patients with disturbances in consciousness by analyzing nursing records. Observations from clinical nurses as well as patients' responses about their care were selected from nursing records. Nursing records from one week of patient care were examined for patients who were unable to speak during hospitalization within a neuro-surgery ward of the hospital. Selected records were classified into the following eight categories: results from monitoring; results from observation; opening and moving the eyes to stimulus; movement of the limbs to stimulus; vocalization to stimulus; facial expression to stimulus; patient's response to care; and miscellaneous. Patients comprised two groups. One group encompassed eight patients with Japan Coma Scale (JCS) II and the other was a group of eight patients with JCS III. When nurses use the JCS to assess patients with disturbances in consciousness, patients who awaken to stimulus are classified as JCS II, while those who do not are JCS III. The total nursing records selected for JCS II were 1551 and 1160 for JCS III. The category of "results from monitoring" was the most selected category within nursing records and accounted for $42.8 \%$ of the JCS III group, while "results from observation" accounted for $38.4 \%$ of the JCS II group. Furthermore, results indicated that the categories of "results from monitoring", "results from observation", and "movement of the limbs for stimulus" had peaked after two to three days, and then abruptly decreased. There were only a few records for the categories of "vocalization to stimulus" and "facial expression to stimulus”, both for the JCS II and the JCS III groups. Even though patients could not verbally indicate their intentions due to problems with consciousness, it is essential for nurses to pay careful attention to the details of patients' reactions.
\end{abstract}

Keywords: Patients; Disturbances in

Consciousness; Nurses; Observation; Nursing Records

\section{INTRODUCTION}

Nursing provides great support for health-related needs. Communication is the process that gives meaning to the content and the relation of messages. Communication is a key basis of nursing because communication plays an important part in understanding patients, winning their confidence, sympathizing with them, and supporting their needs. The sender exchanges thoughts with the receiver, and both interact with one another. However, when nurses care for patients with disturbances in their consciousness, communication may be unclear and difficult to decipher. This may be the reason why nurses feel uneasy, and confusion is not rare in these circumstances.

Therefore, by accurately assessing a patient's level of consciousness, the nurse is able to detect neurological changes and communicate with the medical staff to initiate prompt action, in order to improve survival outcomes and minimize long-term sequelae [1]. Therefore, studies of patients with consciousness disturbances have been investigated in order to verify the reliability and accuracy of assessment tools for assessing various levels of consciousness such as the Glasgow Coma Scale and the National Institutes of Health Stroke Scale [2-8].

In Japan, the previous studies investigating patients with consciousness disturbances mainly focused on verifying the validity of nursing care aimed at life assistance, functional improvement, and consciousness recovery [9-11].

Studies that have developed a new consciousness evaluation scale [12], and analyzed it for use within different situations [13] have been conducted.

Moreover, although the viewpoints of nursing assessments during consciousness level have been investigated $[14,15]$, there have been only a few studies which have 
investigated observations from nurses who were performing these observations to grasp the concept of nursing.

In Japan, nursing records are regarded as one of a nurse's chief duties. Nursing records show a nurse's enforcement and judgment and should reflect nursing practice and methodology. It was expected that an analysis of nursing records (a nurse's thoughts, judgments, and observations) could clarify patient status.

Thus, the objective of the present study was to clearly define clinical nurse's observations of patients with consciousness disturbance through the analysis of nursing records.

\section{METHODS}

\subsection{Sample}

The nursing records for one week from patients who could not speak during hospitalization within a neurosurgery ward of a hospital. Patients were classified into two groups: a group of eight patients with Japan Coma Scale (JCS) II and a group of eight patients with JCS III. Nursing records were specified by nurses from January of 2008 to April of 2012.

\subsection{Data Collection}

The nursing records indicated within the period were chosen from patient ID numbers at random. All data were collected in 2012.

\subsection{Ethical Considerations}

The ethics committee at Hirosaki University Graduate School of Medicine provided ethical approval and the study was registered. We informed potential participants about the study through poster printouts explaining the contents of the study, aspects of confidentiality and that the study was strictly voluntary. Study posters were placed in hospitals within the neuro-surgery ward and in outpatient clinics.

\subsection{Analysis}

We selected observations and patient's responses to their care from nursing records. Selected records were classified into eight categories.

We denoted the eight categories as follows: results from monitoring, results from observations, opening and moving the eyes to stimulus; movement of the limbs to stimulus; vocalization to stimulus; facial expression to stimulus; patient's response to care; and miscellaneous vocalization. We referred to the previous literature for guidance in constructing the category names [15] as well as using the Kohnan score [16,17]. We totaled these records and compared the number of categories observed for one week, in them JCS II with JCS III. All statistical analyses were performed using the Statistical Package for Social Science 12.0 for Windows and the level of significance used was $p<0.05$.

\section{RESULTS}

\subsection{Patient Characteristics}

Table 1 shows the patient characteristics. The main diagnosis of the patients was a subarachnoid hemorrhage or a cerebral hemorrhage. The average age of the patients was $63.4 \pm 19.8$ for the JCS II group, and $66.4 \pm 22.8$ for the JCS III group. The average number of nurses working on nursing records was $10 \pm 2.0$ people for JCS II, and $8.6 \pm 2.1$ people for JCS III. This difference was not significant.

\subsection{Number of Categories within One Week}

Table 2 shows the number of selected nursing records. The total number of nursing records was 1551 for patients in JCS II, and 1160 for patients in JCS III. According to these nursing records, the category of "results from monitoring" was selected the most and accounted for $42.8 \%$ of the JCS III group, while "results from observations" accounted for $38.4 \%$ of the JCS II group. The category of "results from monitoring" was significantly different between the JCS II and JCS III groups. On the other hand, "results from observation", "opening and moving the eyes to stimulus", "movement of the limbs to stimulus", and "vocalization to stimulus" were more prevalent in the JCS II group.

\subsection{Fluctuation in the Number of Categories}

Table 3 shows the fluctuation in the number of categories for one week. Results from monitoring, results from observation, and movement of the limbs to stimulus had peaked within two to three days (of monitoring), and subsequently decreased. The categories of "opening and moving the eyes to stimulus" and "patient's response to care" had peaked within two to three days, repeatedly increasing and decreasing. There were more records of "vocalization to stimulus" in the JCS II group than in the JCS III group. The fluctuation in records of "vocalization to stimulus" was uniformly maintained. The category "facial expression to stimulus" was rarely recorded for patients in both groups.

\section{DISCUSSION}

\subsection{Number of Categories within One Week}

The present study's results indicated that more patient states were recorded for the JCS II than for JCS III. Even though patients had disturbances in consciousness, it was 
Table 1. Patient characteristics.

\begin{tabular}{cccccccc}
\hline \multicolumn{1}{c}{ JCS III group } & & & JCS II group \\
\hline No & Age & Diagnosis & $\begin{array}{c}\text { Average number of nurses } \\
\text { who recorded patient }\end{array}$ & No & Age & $\begin{array}{c}\text { Average number of nurses } \\
\text { who recorded patient }\end{array}$ \\
\hline 1 & 28 & cerebral hemorrhage & 8 & 1 & 64 & subarachnoid hemorrhage \\
2 & 40 & subarachnoid hemorrhage & 7 & 2 & 80 & subarachnoid hemorrhage \\
3 & 76 & cerebral hemorrhage & 6 & 3 & 83 & cerebellar hemorrhage \\
4 & 61 & brain tumor & 11 & 4 & 15 & head injury \\
5 & 69 & cerebral hemorrhage & 7 & 5 & 69 & cerebral hemorrhage \\
6 & 83 & subarachnoid hemorrhage & 10 & 6 & 58 & cerebral hemorrhage \\
7 & 82 & acute subdural hematoma & 8 & 7 & 85 & subarachnoid hemorrhage \\
\hline & 68 & subarachnoid hemorrhage & 12 & 8 & 77 & cerebral hemorrhage \\
\hline
\end{tabular}

Table 2. Frequency of categories within one week.

\begin{tabular}{ccc}
\hline \multirow{2}{*}{ category } & \multicolumn{2}{c}{ Patient record (Percentage of record: \%) } \\
\cline { 2 - 3 } & JCS III & JCS II \\
\hline Result of monitoring & $497(42.8)$ & $596(20.6)$ \\
Result of observation & $458(39.5)$ & $159(10.3)$ \\
Opening and moving the eyes to stimulus & $18(1.6)$ & $220(14.2)$ \\
Movement of the limbs to stimulus & $107(9.2)$ & $138(8.9)$ \\
Vocalization to stimulus & $7(0.6)$ & $11(0.7)$ \\
Facial expression to stimulus & $4(0.3)$ & $78(5.0)$ \\
Patient's response to care & $54(4.7)$ & $30(1.9)$ \\
Miscellaneous & $16(1.4)$ & $1551(100.0)$ \\
total & $1160(100.0)$ & $*$ \\
\hline
\end{tabular}

$\chi^{2}$ test: ${ }^{*} p<0.05$.

Table 3. Change in the number of categories within one week.

\begin{tabular}{|c|c|c|c|c|c|c|c|c|}
\hline Category & & First day & Second day & Third day & Fourth day & Fifth day & Sixth day & Seventh day \\
\hline \multirow{2}{*}{$\begin{array}{l}\text { Results from } \\
\text { monitoring }\end{array}$} & JCS III & $7.6 \pm 7.3$ & $12.8 \pm 4.7$ & $12.4 \pm 8.3$ & $10.4 \pm 8.3$ & $6.6 \pm 5.4$ & $6.5 \pm 5.5$ & $5.9 \pm 4.6$ \\
\hline & JCS II & $5.0 \pm 3.2$ & $4.9 \pm 4.4$ & $6.3 \pm 5.4$ & $6.9 \pm 5.2$ & $6.8 \pm 4.3$ & $4.4 \pm 3.2$ & $5.8 \pm 5.9$ \\
\hline \multirow{2}{*}{$\begin{array}{l}\text { Results from } \\
\text { observation }\end{array}$} & JCS III & $9.4 \pm 4.8$ & $12.4 \pm 4.3$ & $10.6 \pm 4.2$ & $8.6 \pm 6.9$ & $4.9 \pm 2.6$ & $5.4 \pm 2.9$ & $6.5 \pm 5.1$ \\
\hline & JCS II & $11.8 \pm 4.9$ & $12.1 \pm 6.2$ & $16.1 \pm 8.5$ & $12.4 \pm 6.0$ & $7.1 \pm 4.7$ & $6.6 \pm 4.6$ & $8.9 \pm 5.7$ \\
\hline \multirow{2}{*}{$\begin{array}{l}\text { Opening and moving the } \\
\text { eyes to stimulus }\end{array}$} & JCS III & $0.1 \pm 0.4$ & $0.8 \pm 1.4$ & $0.4 \pm 0.7$ & $0.1 \pm 0.4$ & $0.1 \pm 0.4$ & $0.5 \pm 0.5$ & $0.3 \pm 0.7$ \\
\hline & JCS II & $2.3 \pm 1.8$ & $4.1 \pm 2.6$ & $2.1 \pm 1.9$ & $3.9 \pm 2.0$ & $3.1 \pm 2.5$ & $3.0 \pm 2.7$ & $1.5 \pm 3.1$ \\
\hline \multirow{2}{*}{$\begin{array}{l}\text { Movement of limbs to } \\
\text { stimulus }\end{array}$} & JCS III & $3.0 \pm 3.0$ & $5.4 \pm 4.9$ & $1.6 \pm 1.8$ & $1.4 \pm 1.7$ & $0.4 \pm 0.5$ & $0.9 \pm 1.1$ & $0.8 \pm 1.2$ \\
\hline & JCS II & $5.4 \pm 2.1$ & $6.1 \pm 3.9$ & $2.9 \pm 2.1$ & $4.0 \pm 3.2$ & $3.1 \pm 2.0$ & $3.1 \pm 2.9$ & $3.4 \pm 3.3$ \\
\hline \multirow{2}{*}{ Vocalization to stimulus } & JCS III & $0 \pm 0$ & $0.4 \pm 0.7$ & $0 \pm 0$ & $0.1 \pm 0.4$ & $0.1 \pm 0.4$ & $0.1 \pm 0.4$ & $0.1 \pm 0.4$ \\
\hline & JCS II & $2.1 \pm 2.0$ & $3.0 \pm 2.6$ & $1.9 \pm 3.4$ & $2.9 \pm 3.9$ & $2.5 \pm 3.1$ & $2.4 \pm 3.1$ & $2.3 \pm 2.9$ \\
\hline \multirow{2}{*}{$\begin{array}{l}\text { Facial expression to } \\
\text { stimulus }\end{array}$} & JCS III & $0.1 \pm 0.4$ & $0.1 \pm 0.4$ & $0 \pm 0$ & $0.1 \pm 0.4$ & $0 \pm 0$ & $0.1 \pm 0.4$ & $0 \pm 0$ \\
\hline & JCS II & $0.8 \pm 1.0$ & $0.1 \pm 0.4$ & $0 \pm 0$ & $0.1 \pm 0.4$ & $0.1 \pm 0.4$ & $0 \pm 0$ & $0.3 \pm 0.5$ \\
\hline \multirow{2}{*}{ Patient's response to care } & JCS III & $0.3 \pm 0.7$ & $1.0 \pm 0.5$ & $2.4 \pm 2.6$ & $1.4 \pm 1.8$ & $0.8 \pm 1.2$ & $0.6 \pm 0.9$ & $0.4 \pm 0.5$ \\
\hline & JCS II & $0.5 \pm 0.8$ & $2.1 \pm 2.5$ & $1.9 \pm 1.8$ & $0.8 \pm 1.2$ & $1.4 \pm 2.3$ & $1.4 \pm 2.4$ & $1.8 \pm 2.2$ \\
\hline
\end{tabular}

(n = (8); JCS II ( $\mathrm{n}=8$ ). Split plot design. ${ }^{*} p<0.05$. 
considered that responses for JCS II were more prevalent than for JCS III. Because the patients were diagnosed as seriously ill, nurses had recorded various characteristics, including the patients circulation, respiration, nerve movements, blood pressure, and papillary reflex for the categories of "results from monitoring" and "results from observation". In the present study, it was suspected that medical practice as well as nurses may place great importance on life support methods. It may be assumed that the categories of "opening and moving the eyes to stimulus", and "movement of the limbs to stimulus" were recorded more frequently than the category of "facial expression to stimulus" because a change in a patient's eye movements or limb movement was probably more easily observed than a change in their facial expression.

\subsection{Fluctuation in the Number of Categories}

A peak in the number of nursing records were seen for many of the categories on the second or third day, which corresponds to the acute period when patients took ill and were hospitalized; this is known from a clear change in status in the patient analyzed. Moreover, since there were patients among the analyzed who were hospitalized during the night, it was thought that the number in the contents for the first record decreased compared to the other days.

There were only a few records for the categories of "vocalization to stimulus" and "facial expression to stimulus", both for JCS II and JCS III. Sugimoto et al. [18] stated that when nurses felt that a patient was behaving abnormally, they caught nonverbal signs, and catching these signs and considering their meaning was important. Moreover, accurately reading a patient's minute changes is an important ability for a nurse. Even though patients could not verbally indicate their intentions due to problems with consciousness, it is essential for nurses to pay careful attention to the details of patients' reactions.

\section{LIMITATIONS OF THE REVIEW}

We suspected that nurses may not have recorded everything they observed. Because it is evident that nurses have certain cues and signs they focus on and pay more attention to, a more detailed study of nursing records is necessary.

\section{CONCLUSIONS}

In order to study clinical nurses' observations of patients with consciousness disturbance, we analyzed nursing records of patient progress.

Nursing records were indicated more for patients with JCS II than for patients in JCS III. "Results from monitoring" and "results from observation" were found to be the most recorded categories in nursing records.

\section{REFERENCES}

[1] Waterhouse, C. (2005) The Glasgow Coma Scale and other neurological observations. Nursing Standard, 19, 56-64. http://dx.doi.org/10.7748/ns2005.04.19.33.56.c3857

[2] Juarez, V.J. and Lyons, M. (1995) Interrater reliability of the Glasgow Coma Scale. Journal of Neuroscience Nursing, 27, 283-286. http://dx.doi.org/10.1097/01376517-199510000-00008

[3] Dewey, H.M., Donnan, G.A., Freeman, E.J., Sharples, C.M., Macdonell, R.A. and McNeil, J.J. (1999) Interrater reliability of the National Institutes of Health Stroke Scale: Rating by neurologists and nurses in a communitybased stroke incidence study. Cerebrovascular Disorders, 9, 323-327. http://dx.doi.org/10.1159/000016006

[4] Joao, F.F., Simoes, L., Luis, M.T., Jesus, D.V., Sa-Couto, P., Fernandes, J. and Morgado, M. (2011) Assessment of comatose patients: A Portuguese instrument based on the coma recovery scale-revised and using nursing standard terminology. Journal of Advance Nursing, 65, 1129-1141.

[5] Cheung, R.T.F., Lyden, P.D., Tsoi, T.H., Huang, Y., Liu, M., Hon, S.F.K., Raman, R. and Liu, L. (2010) Production and validation of putonghua-and cantonese-Chinese Language National Institutes of Health Stroke Scale training and certification videos. World Stroke Organization International Journal of Stroke, 5, 74-79. http://dx.doi.org/10.1111/j.1747-4949.2010.00411.x

[6] Dawes, E., Lloyd, H. and Durham. L. (2007) Monitoring and recording patients' neurological observations. Nursing Standard, 22, 40-45. http://dx.doi.org/10.7748/ns2007.11.22.10.40.c6237

[7] Formisano, R., Carlesimo, G.A., Sabbadini, M., Loasses, A., Penta, F., Vinicola, V. and Caltagirone, C. (2004) Clinical predictors and neuropsychological outcome in severe traumatic brain injury patients. Acta Neurochirurgica, 146, 457-462.

http://dx.doi.org/10.1007/s00701-004-0225-4

[8] Phuping, A. (2009) Endorsement of the four Score for consiciousness assessment in neurosurgical patients. $\mathrm{Neu}$ rologia Medico-Chirurgica, 49, 565-571.

http://dx.doi.org/10.2176/nmc.49.565

[9] Hinoura, Y. and Watanabe, K. (2008) The methods and future tasks nursing intervention for patients in persistent vegetative state. Bulletin of School of Health Sciences Faculty of Medicine, Niigata University, 9, 189-197. (in Japanese).

[10] Kumiko, M. and Yuko, H. (2013) Review of nursing literature on patients with persistent disturbance of consciousness in Japan. Journal of comprehensive nursing research, 14, 3-16. (in Japanese).

[11] Mitsue, S., Kazuo, Y., Hiroshi, K. and Takeshi, K. (2011) Study on evaluation indices for care to the patients with persistent unconsciousness. 3rd Report, Eastern Medicine, 27, 39-49. (in Japanese).

[12] Takahashi, C., Okudera, H., et al. (2007) What is an excellent coma scale? Comparative study of the JCS and 
ECS-first report. Neurosurgical Emergency, 12, 129-135. (in Japanese).

[13] Yoshisaki, M. and Okudera, H. (2010) Current status of clinical evaluation on consciousness level in nursing practice. Journal of Regional Emergency and Disaster Medicine Research, 9, 23-27. (in Japanese).

[14] Shimomura, A. (2004) The indicators of nurses' assessment for judging level of consciousness. Rehabilitation Nursing, 3, 17-25. (in Japanese).

[15] Okubo, N., Shiro, M., et al. (2002) Analysis of clinical nurses' observations of patients with protracted consciousness disturbance-basis for determining improvement of their consciousness level. The Society for Treatment of Coma, 11, 27-32.

[16] Satoru, F., Nobukazu, N., Kuniaki, O., et al. (1993) Eva- luation of the severity of prolonged consciousness disturbances after head injury: A scoring system developed in our department. The Society for Treatment of Coma, 2, 173-183.

[17] Satoru, F., Nobukazu, N. and Yoshihide, N. (1997) Reliability and factorial structure of a rating scale for persistent vegetative state. Brain and Nerve, 49, 1139-1145. (in Japanese)

[18] Sugimoto A., Horikosi M., et al. (2005) Clinical Decision-making by nursing staff according to their perceptions of patients' abnormalities. The Kitakanto Medical Journal, 55, 123-131. (in Japanese). http://dx.doi.org/10.2974/kmj.55.123 